\title{
Metastatic meningioma: The role of whole-body diffusion-weighted imaging
}

\author{
TERESA CABADA $^{1}$, REBECA BERMEJO ${ }^{1}$, CARMEN BACAICOA $^{1}$ and ANA MARTÍNEZ-PEÑUELA ${ }^{2}$ \\ Departments of ${ }^{1}$ Radiology, and ${ }^{2}$ Pathology, Hospital de Navarra, Pamplona 31008, Spain
}

Received January 16, 2011; Accepted June 9, 2011

DOI: $10.3892 / \mathrm{ol} .2011 .329$

\begin{abstract}
We report the case of a 74-year-old male patient with a completely resected anaplastic meningioma who developed multiple metastases two years later (subcutaneous tissue near the surgical area, cervical lymph nodes, lung, pleura and bones). The primary tumor and all of the metastases showed a significant restricted diffusion. Whole-body diffusion-weighted imaging (DWI) was performed for assessment of the metastases. This case demonstrated the usefulness of this technique in screening extracranial metastases in patients with malignant meningiomas.
\end{abstract}

\section{Introduction}

Extracranial metastases of meningioma is a rare but well documented event. Its incidence has been estimated to account for less than $0.1 \%$ of intracranial meningiomas. The most frequent sites for metastases are the lungs, liver, bones and cervical lymph nodes. Remote metastases can occur in histologically benign and atypical or malignant meningiomas, with variable disease-free periods (1-6).

Diffusion-weighted imaging (DWI) provides a new contrast mechanism for the evaluation of tumors by imaging the microscopic motion of water molecules. The appearance of meningiomas in DWI is variable, but they usually show diffusion values similar to the brain parenchyma. Malignant meningiomas exhibit lower values in apparent diffusion coefficient (ADC) maps than those of benign tumors. However, the usefulness of this DWI for predicting the histological grade remains controversial (7).

Whole-body DWI was developed as a useful technique for cancer imaging, and can be used for improving tumor detection, characterizing certain tumors and monitoring response to treatment. DWI is an efficacious method of detecting osseous and extraosseous metastases (8-11).

Correspondence to: Dr Teresa Cabada, Department of Radiology, Hospital de Navarra, C/Irunlarrea 3, Pamplona 31008, Spain

E-mail: tcabadag@hotmail.es

Key words: meningioma, metastases, magnetic resonance imaging, diffusion-weighted imaging
We report the case of a patient with a malignant meningioma with significant restricted diffusion and multiple metastases. We emphasize the value of the whole-body DWI for screening extracranial metastases in these tumors.

\section{Case report}

A 74-year-old male was admitted to hospital with partial seizures and headaches. Computed tomography (CT) and magnetic resonance imaging (MRI) showed a right frontal dural-based tumor with skull infiltration and a low signal in the ADC map. The patient underwent a complete removal of the tumor and received radiotherapy.

Microscopic examination showed a densely cellular tumor composed of sheets and whorls of cells with ample eosinophilic cytoplasm and oval to round vesicular or hyperchromatic nuclei, generally with large nucleoli. Frequent mitotic figures were observed along with moderate pleomorphism and foci of necrosis. Tumor-invaded bone and cerebral parenchyma were also evident. Immunohistochemistry showed the tumor cells to be positive for epithelial membrane antigen (EMA) and vimentin. The MIB-1 (Ki67) labeling index was approximately 25\%. The diagnosis was anaplastic meningioma (WHO grade III) (Fig. 1).

Two years later the patient complained of shoulder pain and an MRI showed a right laterocervical mass with osteolysis of C6 and braquial plexus infiltration (Fig. 2). The apperance of a new nodule in the subcutaneous tissue near the surgical scar was also noted. Cranial MRI showed a small local recurrence and a subcutaneous lesion. The two lesions showed extremely low values on the ADC map, similar to the original tumor, and they were biopsied. Microscopic examination and an immunohistochemistry study showed a malignant tumor with similar features to that of the primary anaplastic meningioma (Fig. 2).

A whole-body MRI with DWI was performed in order to assess metastatic disease. DWI was acquired with echo-planar imaging combined with parallel imaging and fat suppression (repetition time/echo time, 3900/76 sg; matrix 192, field of view 380, $b=50-400-800)$. Multiple lesions with restricted diffusion were found in the spine, ribs, sacrum, pelvis and thorax (pulmonary and pleural lesions). Bone lesions were confirmed in T1 and STIR sequences and lung lesions were confirmed by CT. The CT also detected more lung nodules of small size (Fig. 3). 


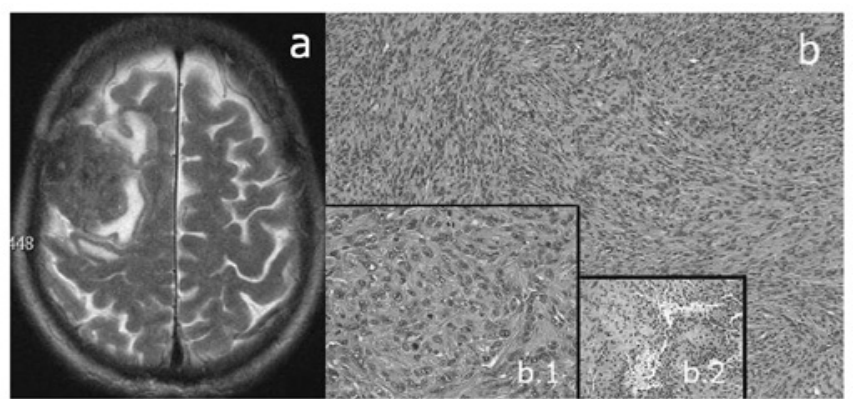

Figure 1. (A) Axial T2-weighted image shows a right frontal extraxial mass. (B) The histopathological sections show a densely cellular tumor composed of sheets and whorls of cells with ample eosinophilic cytoplasm; (b.1) round to oval vesicular nuclei with large nucleoli, atypia and frequent mitotic figures, and (b.2) marked cellular pleomorphism and foci of geographic necrosis.

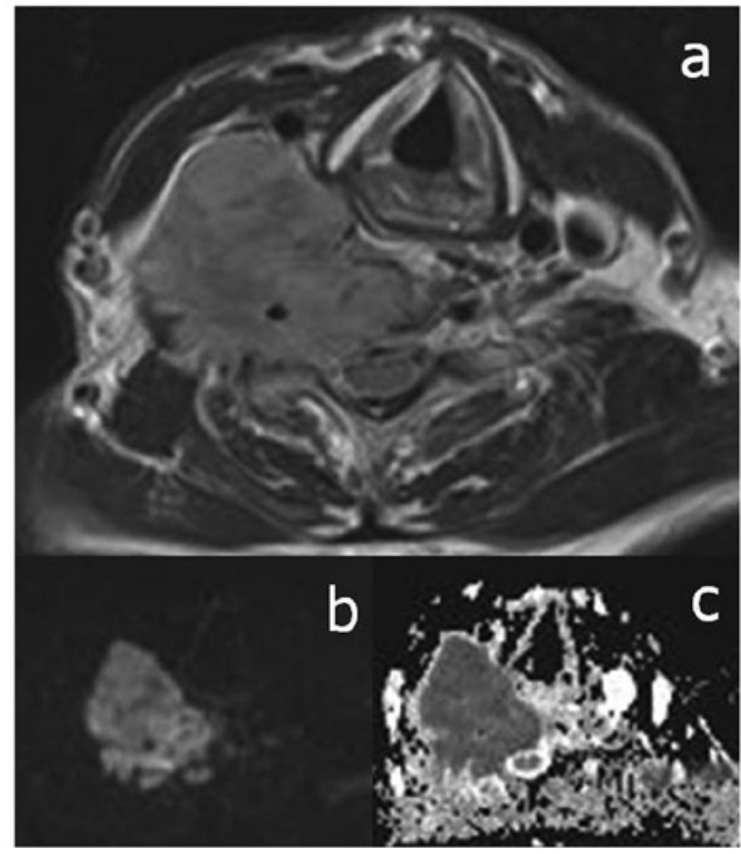

Figure 2. (A) Axial T2-weighted image in the neck. (B) DWI, b=1000 and (C) ADC map. A soft tissue mass in the right side with bone infiltration is shown. The mass exhibits a significant restricted diffusion.

The patient received chemotherapy and palliative radiotherapy with a poor outcome. He succumbed to the disease six months after therapy was administered.

\section{Discussion}

Metastatic spreading is a rare complication of meningiomas. Numerous reports are available regarding metastases from benign and malignant meningiomas (2-6). Meningiomas occur more frequently in atypical and malignant tumors (WHO grade II and III), particularly multiple metastases $(1,3,4)$.

The most frequent site of metastases is the lung, where lesions can be solitary (more usual with benign meningiomas) (2) or multiple $(3,4)$. However, metastases also occur in the pleura (4). Bone is another metastatic target, in particular the spine (3). Our patient presented multiple metastases in the

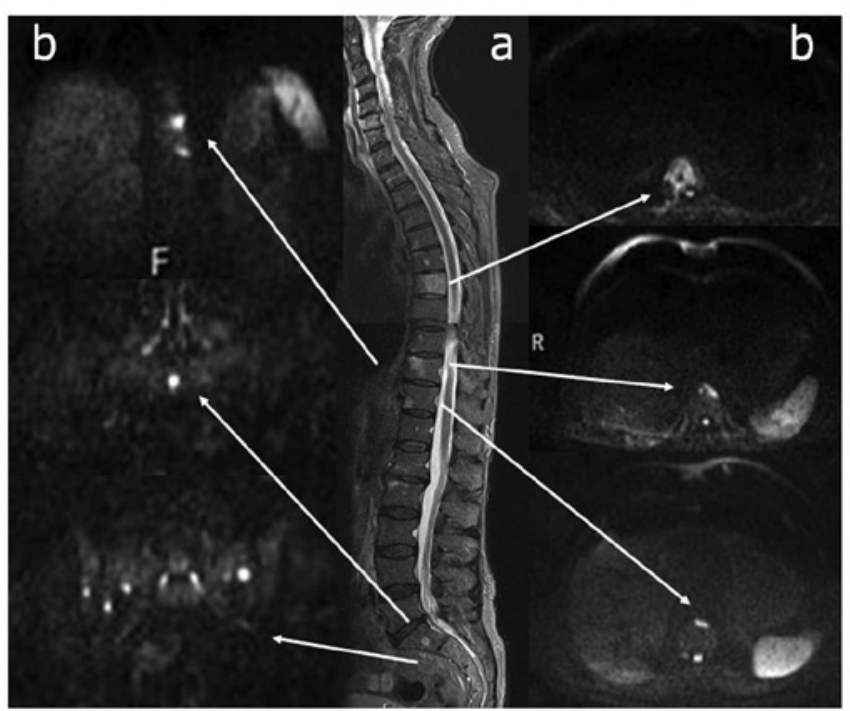

Figure 3: (A) Composed sagittal STIR image of the spine. (B) DWI, b=800, coronal (left) and axial (right) images at different levels show multiple bone metastases in vertebra, sacrum and pelvis.

lungs, pleura and bones (including the spine, ribs and sacrum), and in the cervical lymph nodes.

Meningiomas are capable of disseminating via hematogeneous, lymphatic or cerebrospinal fluid pathways. Hematogeneous metastasis possibly occurs most frequently as a result of the occasional invasion of the venous sinuses and large vessels. This invasion explains the high incidence of lung and liver metastases. Batson's plexus, a common pathway for vertebral metastases, is associated with intracranial and cranial venous circulation.

Metastases can also be found after surgery as a iatrogenic spreading in the operative route. They have been reported in the subcutaneous tissue, periostium and as spinal drop metastases through the cerebrospinal fluid route, in both benign and malignant meningiomas $(5,6)$. The subcutaneous lesion in the case reported may be interpreted as secondary to iatrogenic spreading, considering its proximity to the surgical area.

DWI provides functional information and can be used for the detection and characterization of pathological processes including malignant tumors. It is based on the molecular mobility of water. This mobility is determined by the interaction of water with intracellular elements, macromolecules, cell membranes, cell density and microstructural organization (7-10).

DWI is a well-established method of examining the brain. Extracranial DWI, however, did not become a clinical standard as the use of echo-planar imaging was complicated by magnetic susceptibility artefacts, poor anatomic detail, and severe image distortion in the body. Recently introduced parallel imaging techniques and the development of stronger gradients and multichannel coils have partially overcome these problems. These advances enable the technique to be widely applied for tumor evaluation in bones, the abdomen and the pelvis, and have led to the development of the whole-body DWI (8-10).

Evaluation of areas close to the heart and the diaphragm is limited due to signal loss and motion artefacts. These 
obstacles explain the limited value of this technique for detecting lung metastases in our patient.

One of the challenges to the widespread adoption of DWI in the assessment of body tumors is the lack of standardization, including the choice of $b$ values. Low $b$ values are selected in certain studies to allow for more T2 contrast (10).

Primary tumors and metastases exhibiting restricted diffusion are identified with DWI (8-11). Whole-body DWI shows similar values to scintigraphy or PET/CT when screening bone metastases of prostate and breast cancer $(10,11)$.

To the best of our knowledge, the use of DWI in the assessment of metastases from intracranial tumors, including meningiomas, has not been reported previously. In these infrequent cases, particularly in malignant meningiomas with restricted diffusion, the use of this technique may be considered a standard clinical protocol for metastasis screening.

In conclusion, metastasizing meningioma is a rare event occurring mainly in malignant tumors. Whole-body DWI can be used for the assessment of metastases in patients with malignant meningioma.

\section{References}

1. Perry A, Louis DN, Scheitahuer BW, Budka H and von Deimling A: Meningeal tumours. In: Louis DN, Ohgaki H, Wiestler OD, Cavenee WK (eds). World Health Organisation Classification of Tumours of the Central Nervous System. IARC Press, Lyon, pp163-186, 2007.

2. Fulkerson DH, Horner TG and Hattab EM: Histologically benign intraventricular meningioma with concurrent pulmonary metastasis: case report and review of the literature. Clin Neurol Neurosurg 110: 416-419, 2008
3. Lee GC, Choi SW, Kim SH and Kwon HJ: Multiple extracranial metastases of atypical meningiomas. J Korean Neurosrg Soc 45: 107-111, 2009.

4. Erman T, Hanta I, Haciyakupoglu S, Zorludemir S, Zeren H and Göçer AI: Huge bilateral pulmonary and pleural metastasis from intracranial meningioma: a case report and review of the literature. J Neurooncol 74: 179-181, 2005.

5. Akai T, Shiraga S, Izuka H, Kishibe M, Kawakami S and Ueda Y: Recurrent meningioma with metastasis to the skin incision. Case report. Neurol Med Chir (Tokyo) 44: 600-602, 2004.

6. Kuroda H, Kashimura H, Ogasawara K, Sugawara A, Sasoh M, Arai $\mathrm{H}$ and Ogawa A: Malignant intracranial meningioma with spinal metastasis. Case report. Neurol Med Chir (Tokyo) 49: 258-261, 2009.

7. Cabada T, Caballero MC, Insausti I, Alvarez de Eulate N, Bacaicoa C,Zazpe I and Tuñón T: The role of difusión-weighted imaging in the evaluation of meningiomas: radio-pathologic correlation. Radiología 5: 41-49, 2009.

8. Koh DM and Collins DJ: Diffusion weighted MRI in the body: applications and challenges in oncology. AJR Am J Roentgenol 188: 1622-1635, 2007.

9. Kwee TC, Takahara T, Ochiai R, et al: Whole body diffusion weighted magnetic resonance imaging. Eur J Radiol 70: 409-417, 2009.

10. Luboldt W, Küfer R, Blumstein N, Toussaint TL, Kluge A, Seemann MD and Luboldt HJ: Prostate carcinoma: diffusion weighted imaging as potencial alternative to conventional MR and 11-C choline PET/CT for detection of bone metastases. Radiology 249: 1017-1025, 2008.

11. Gutzeit A, Doert A, Froehlich JM, et al: Comparison of diffusion weighted whole body MRI and skeletal scintigraphy for the detection of bone metastases in patients with protate or breast carcinoma. Skeletal Radiol 39: 333-343, 2010. 